\title{
Vertical coupling of the middle atmosphere during stratospheric warming events
}

\author{
Andreas Miller $^{\mathrm{a}, \mathrm{b}, *}$, Hauke Schmidt $^{\mathrm{a}}$, Felix Bunzel ${ }^{\mathrm{a}}$ \\ ${ }^{a}$ Max Planck Institute for Meteorology, Bundesstr, 53, D-20146 Hamburg, Germany \\ b Department of Earth, Atmospheric and Planetary Sciences, 1715 Massachusetts Institute of Technology, 77 Massachusetts Avenue, Cambridge, \\ MA 02139-4307, USA
}

\section{A R T I C L E I N F O}

\section{Article history:}

Received 6 August 2012

Received in revised form

18 January 2013

Accepted 7 February 2013

Available online 24 February 2013

Keywords:

Sudden stratospheric warmings

Mesospheric coolings

Vertical couplings

Middle atmosphere dynamics

\begin{abstract}
A B S T R A C T
A 20 year simulation of the high-top atmospheric General Circulation Model (GCM) HAMMONIA is used to investigate internally produced Sudden Stratospheric Warmings (SSWs). We detect 19 major SSWs and evaluate the model results by comparison to the ERA40 reanalysis dataset. Composites are built to analyze the climatological characteristics of SSWs, in particular to investigate the mesospheric precursors and differences between vortex splits and displacements. The vertical coupling processes from the stratosphere to the lower thermosphere are studied using transformed Eulerian-mean (TEM) analysis and diagnostics concerning the role of gravity waves. Consistent to recent studies, we find a mesospheric cooling and a weaker thermospheric warming accompanying the SSW. The large anomalies in the zonal mean winds and temperatures are explained by the interactions of EP-Flux divergence, mean flow advection and parameterized momentum deposition of gravity waves. We present an algorithm, based on geopotential height, to classify the events. Nine SSWs can be characterized as vortex splits, 10 as displacements. The differences between the two types are statistically significant suggesting splits are associated with larger anomalies in temperature and zonal wind. Investigation of the longitudinal dependence of zonal winds demonstrates the asymmetry of the climatological winter and of single events. Therefore, we do not find a criterion to sufficiently detect SSWs using mesospheric winds prior to the central date.
\end{abstract}

(c) 2013 Elsevier Ltd. All rights reserved.

\section{Introduction}

Upward propagating planetary waves over arctic winter regions occasionally lead to a significant deceleration of the mean zonal winds in the stratosphere, which are associated with significant warming rates over time periods ranging from days to weeks (Matsuno, 1970). These sudden stratospheric warmings (SSWs) were first described by Scherhag (1952). Matsuno (1971) first explained their connection to tropospheric Rossby waves. In this study we focus only on major SSWs (from now on just SSWs) as described by Charlton and Polvani (2007). SSWs can be associated with significant displacements or complete breakups of the polar vortex. In recent years, observational (Baldwin and Dunkerton, 2001) and model studies (Polvani, 2002) confirmed that the stratosphere is not only responding to tropospheric

\footnotetext{
* Corresponding author at: Present address: Department of Earth, Atmospheric and Planetary Sciences, 1715 Massachusetts Institute of Technology, 77 Massachusetts Avenue, Cambridge, MA 02139-4307, USA. Tel.: +1 617253 3715; fax: +1 16172538298 .

E-mail addresses: andreas.miller@zmaw.de (A. Miller), hauke.schmidt@zmaw.de (H. Schmidt), felix.bunzel@zmaw.de (F. Bunzel).
}

forcings, but that coupling mechanisms act in both ways. As SSWs are the most prominent demonstration of connections of lower, middle and upper atmosphere over arctic regions, they are crucial to our understanding of interactions among the different atmospheric layers (Charlton and Polvani, 2007).

A number of studies describe the occurrence of a mesospheric cooling accompanied with SSWs (Smith, 1996; Liu and Roble, 2002; Hoffmann et al., 2007; Siskind et al., 2007). Some of them used observations to study single events. Others studied the results obtained from model simulations that were either nudged to reproduce observed SSWs or, less often, produced SSWs internally. It has been noted that the mesospheric cooling is likely to precede the stratospheric warming by a few days (Liu and Roble, 2002; Hoffmann et al., 2007). The studies of Liu and Roble (2002), Fuller-Rowell et al. (2010) and Goncharenko et al. (2010) also presented hints for thermospheric and ionospheric signals during SSWs, but, in this study, we will focus on dynamical processes below $10^{-4} \mathrm{hPa}$.

Despite the ongoing research over several decades, many questions remain unsolved. As most datasets are limited by either the number of events or by the spatial and temporal coverage of observations, it is not well known how much their results depend 
on the particular event or the specific observational site. Consequently, it is difficult to compare zonal mean values, which define SSWs, to point measurements, which motivate the idea of mesospheric precursors. Hence, the question remains if the occurrence of mesospheric coolings or wind reversals can be used as a predictor for SSWs.

Another focus of recent studies was on the differences between SSWs that were dominated by wavenumber 2 (split) or wavenumber 1 (displacement). Although Yoden et al. (1999) and recently Charlton and Polvani (2007) found dynamical differences between these types in the stratosphere and below little is known about possible corresponding structures at higher altitudes.

In this study, we are filling the gap between high reaching single case studies on the one side and model composites, which, with the exception of Yoden et al. (1999), usually did not cover altitudes above the stratopause. For this purpose, we analyze a 20-year model run of the high atmosphere resolving Global Circulation Model (GCM) HAMMONIA (Hamburg Model of the Neutral and Ionized Atmosphere). Although we evaluate the quality of our experimental dataset carefully, all model results are limited by the capability of the model to produce a realistic climate.

The internal variability of SSWs in the model is investigated by building composites and calculating anomalies with respect to the model climatology. These experiments provide the opportunity to study the vertical structure of 19 SSWs from the surface up to the thermosphere. The simulated SSWs are compared to reanalysis data and other model studies to evaluate the quality of the performed experiment.

The composites of SSWs are then used to study:

- the vertical coupling processes during SSWs from the tropopause to about $10^{-4} \mathrm{hPa}$

- the dynamical differences between vortex splits and displacements in the middle atmosphere

- if mesospheric precursors can be identified and whether they allow to predict SSWs

The study is structured as follows: In Section 2, we describe the HAMMONIA model, the dataset produced by it and postprocessing methods to investigate the issues mentioned above. Sections 3 and 4 present and discuss the results, which lead to the conclusions in Section 5.

\section{Data and methods}

\subsection{Model and data}

We are using HAMMONIA, a general circulation and chemistry model covering the atmosphere from the surface up to the lower thermosphere (Schmidt et al., 2006). HAMMONIA is an upward extension of the ECHAM5 GCM (Roeckner et al., 2006; Manzini et al., 2006) coupled to the chemical scheme of MOZART3 (Model of Ozone and Related Tracers; Kinnison et al., 2007). The model has a spectral dynamical core with triangular truncation at wave number 31 (T31), corresponding to a Gaussian grid of $96 \times 48$ grid points (i.e. a mesh size of $3.75^{\circ}$ ). For this study a model version with 67 vertical layers from the surface up to $1.7 \times 10^{-7} \mathrm{hPa}$ $(\approx 250 \mathrm{~km})$ is used. The distance between the layers above the tropopause is roughly $2-3 \mathrm{~km}$. Due to this coarse vertical resolution the model does not internally produce a quasi-biennial oscillation of stratospheric winds (QBO). The simulation analyzed here is described in more detail by Schmidt et al. (2006). It is a time slice simulation using climatological prescribed sea surface temperatures, green house gas (GHG) concentrations representing the 1990s and solar irradiance representative for permanent solar minimum conditions as boundary conditions. The model is not forced to match observations in the lower atmosphere and therefore does not compare to 20 calendar years in the past. However, it does represent a model climatology with internally produced variability. More recent model simulations use higher vertical resolutions, in particular in the upper troposphere and the stratosphere (up to 119 levels), and thereby are capable of internally producing a QBO (Schmidt et al., 2010). Both vertical resolutions lead to a realistic number of SSWs, but the more realistic frequency and seasonal cycle are produced by using more vertical levels. In this study, we analyze the less recent simulation because of the availability of model output with a high temporal resolution ( 3 hourly) also for diagnostic parameters as momentum deposition of gravity waves. In the model, subgrid-scale gravity waves are parameterized according to the schemes of Lott and Miller (1997) for orographic sources, and Hines (1997a,b) for non-orographic sources. A more detailed model description is provided by Schmidt et al. (2006).

\subsection{Methods}

We identify stratospheric warmings between October and April using the same criteria as Charlton and Polvani (2007) while considering the corrigendum to the publication (Charlton-Perez and Polvani, 2011). These criteria are fulfilled when the zonally averaged zonal wind at $60^{\circ} \mathrm{N}$ on the $10 \mathrm{hPa}$ pressure surface changes its direction from west to east. The first occurrence of easterly winds is called the central date of the SSW. Another SSW is only detected after at least 20 consecutive days of westerly zonal mean winds. Final warmings are excluded by the condition that the wind has to turn back to westerly for at least 10 consecutive days before April 30th. According to the criteria defined by the World Meteorological Organization (WMO), the detection of a major warming would also require a reversal of the horizontal temperature gradient, in addition to the reversal of the mean zonal wind. This temperature criterion is fulfilled if the difference between the zonal mean temperature at $60^{\circ} \mathrm{N}$ and the North Pole turns positive. Similarly to Charlton and Polvani (2007) we ignore this criterion as it has only a small influence on the results of the detection algorithm. Our analysis leads to the identification of 19 warmings not equally distributed over the 20 winter seasons (see Table 1 ).

In a second step, we categorize the events as splits and displacements, as suggested by Charlton and Polvani (2007) and Yoden et al. (1999). Unfortunately, there is no commonly accepted definition for these categories. Most studies used criteria based on either the zonal wavenumber (Yoden et al., 1999) or on different forms of vorticity gradients (Charlton and Polvani, 2007). For simplicity reasons, we are applying an algorithm that analyzes geopotential height at the $10 \mathrm{hPa}$ level. If the zonal wind reverses, the algorithm determines if more than one minimum in geopotential height exists and if the minima are sufficiently disconnected to indicate a vortex split. A detailed description of the algorithm is given in Appendix A. The algorithm was evaluated using ERA40 reanalysis data, interpolated to T31, for which it delivers similar results as the identification method presented by Charlton and Polvani (2007) and agreed well with subjective impressions. In Section 3.3, the differences between splits and displacements are further analyzed using the 'Test for Differences of Mean under Independence' as described by Wilks (2006).

In order to test if differences among the commonly used categories of SSWs and mesospheric precursors are general features of SSWs or occur only under certain circumstances or at certain locations, we compile composites of SSWs. We limit our 
Table 1

SSWs as detected in the HAMMONIA experiment. Years represent the number of the 20 year run, but do not refer to actual calendar dates. $\Delta T_{10}$ shows the mean polar cap temperature anomaly at $10 \mathrm{hPa} \pm 5$ days around the central date.

\begin{tabular}{|c|c|c|c|}
\hline No. & Central date & Type & $\Delta T_{10}(\mathrm{~K})$ \\
\hline 1 & 8 Nov 01 & Displacement & 3.1 \\
\hline 2 & 14 Mar 02 & Displacement & 7.6 \\
\hline 3 & 21 Mar 03 & Split & 1.3 \\
\hline 4 & 4 Dec 03 & Displacement & 4.3 \\
\hline 5 & 25 Feb 06 & Split & 4.7 \\
\hline 6 & 9 Feb 08 & Displacement & 4.4 \\
\hline 7 & 27 Nov 08 & Displacement & 2.1 \\
\hline 8 & 27 Mar 10 & Split & 1.1 \\
\hline 9 & 3 Nov 10 & Displacement & 2.4 \\
\hline 10 & 27 Jan 11 & Split & 0.6 \\
\hline 11 & 22 Mar 11 & Displacement & 1.3 \\
\hline 12 & 24 Feb 13 & Split & 2.5 \\
\hline 13 & 5 Nov 14 & Split & 3.0 \\
\hline 14 & 23 Mar 15 & Displacement & -0.9 \\
\hline 15 & 27 Feb 16 & Split & 10.4 \\
\hline 16 & 22 Nov 16 & Displacement & 3.6 \\
\hline 17 & 17 Feb 17 & Displacement & 6.3 \\
\hline 18 & 24 Dec 18 & Split & 16.3 \\
\hline 19 & 11 Feb 19 & Split & -0.7 \\
\hline
\end{tabular}

analysis to the period of 31 days around the central date of a SSW (from now on simply referred to as 'event'). Recent studies have often chosen periods between one and two months to study SSWs (Charlton and Polvani, 2007; Hoffmann et al., 2007). The choice of 31 days is therefore arbitrary to some degree but seems to be appropriate for our purposes. The composites are computed as the unweighted average over all events.

In this study, the climatological winter is computed as the average over the 20 winter seasons (from October 1st to April 30 th) of the model run resulting in a mean state, which accounts for the seasonal cycle over the seven month period. The time and height dependent anomalies, which are used for the plots in Sections 3.2 and 3.3, are calculated to point out the prominent nature of SSWs compared to the relatively undisturbed mean winter state of the stratosphere. Furthermore, they avoid the large range of many variables over the extended altitude range covered by HAMMONIA. We define anomalies as the difference of the 31 day time series of an event to the same period of the climatological winter. Thus, the SSWs are compared to the climatology, which the model produces during the time of the year when the event is detected. Note that the winter mean state is time dependent as it includes the seasonal cycle. In Figs. 3a and 4a, we use the standard deviation of the composite members to describe the spread of the distribution. The shaded areas in these figures indicate values of the composite anomaly, which deviate from the climatological winter by more than one standard deviation.

The analyzed variables are geopotential height, temperature, wind, momentum deposition due to gravity waves and the different quantities, that result from a Transformed Eulerian Mean (TEM) calculation. The TEM was performed following the derivation of Andrews et al. (1987).

\section{Results and discussion}

\subsection{Evaluation of model results}

The investigated experiment contains 19 SSWs in 20 years (see Table 1), which translates to a ratio of 0.95 events per year. We compare our results to the analysis of ERA-40 data in Charlton and Polvani (2007) which results in 0.64 events per year between
1 September 1957 and 31 August 2002 (29 SSWs). Charlton and Polvani (2007) also report similar numbers for the NCEP-NCAR reanalysis, but, for the purpose of this paper, we will focus on ERA-40. The interdecadal variability in ERA-40 is high, and from the mid 1960s to 1973 as well as during the 1980s the frequency of SSWs is close to 1 event per year. The ratio of vortex displacements to vortex splits in our simulation is 1.11 and thereby almost equal to the averaged value of 1.10 given by Charlton and Polvani (2007).

One measure for the intensity of a SSW is the characteristic temperature anomaly $\left(\Delta T_{10}\right)$ listed in the last column of Table 1. Following Charlton and Polvani (2007), we calculate the polar cap temperature anomaly as the area-weighted mean temperature anomaly North of $50^{\circ} \mathrm{N}$ and at $10 \mathrm{hPa}$. The intensity measure $\left(\Delta T_{10}\right)$ is then defined as the average over these polar cap temperature anomalies from 5 days before to 5 days after the central date. The numbers help to evaluate our model results as they can easily be compared to the mentioned ERA40 analysis of Charlton and Polvani (2007).

Although the range of temperature anomalies in our dataset is close to the range in the observations (from $-0.9 \mathrm{~K}$ to $16.3 \mathrm{~K}$ and from $-2.9 \mathrm{~K}$ to $14.1 \mathrm{~K}$ respectively), the average over all SSWs is lower (3.7 K in HAMMONIA, 8.2 K in ERA40). However, our result is still within one standard deviation of the observational mean. As in the case of reanalysis data, the simulated warmings are, on average, stronger for splits than for displacements. More details about the anomalies and the differences between the types of events are presented in Sections 3.2 and 3.3 respectively.

Figs. 1 and 2 compare composites of SSWs of the zonally averaged zonal wind and temperature from (a) HAMMONIA and (b) ERA40, which are only available up to $1 \mathrm{hPa}$. The model output is plotted in $3 \mathrm{~h}$ intervals, the reanalysis data are shown as daily means. As the vertical coordinate is pressure for both the model and the reanalysis data, the log-pressure system is used to provide altitude information.

The time evolution of the HAMMONIA zonal winds at $60^{\circ} \mathrm{N}$ agrees fairly well with the reanalysis. Both plots show westerly winds from the surface up to the top of the ERA40 dataset during the first 14 days of the analyzed time frame. However, the westerly winds at the beginning of the composite are about $15 \mathrm{~ms}^{-1}$ stronger in ERA40 (Fig. 1b) as they are in HAMMONIA (Fig. 1a). The maximum easterly wind speeds around the central date are also larger in the reanalysis data. This indicates that the intensity of SSWs is underestimated by the model. By definition, the mean zonal winds turns easterly on day 15 at $10 \mathrm{hPa}$. The zonal wind changes sign almost simultaneously between $10 \mathrm{hPa}$ and $1 \mathrm{hPa}$ in both the model and the reanalysis. The occurrence of westerly winds above $20 \mathrm{~ms}^{-1}$ in ERA40 during the end of the analyzed period of time could be interpreted as a shorter recovery phase in the reanalysis data than in HAMMONIA. In the upper mesosphere (around $10^{-2} \mathrm{hPa}$ ), the model simulates the characteristic easterlies before the SSW occurs. Approximately one week before the central date, these winds start to propagate downwards leading to a broad band of easterlies between 10 and $10^{-3} \mathrm{hPa}$ for about 3-4 days around the central date. The dynamical changes in the mesosphere prior to the onset of the SSW agree with recent studies about mesospheric precursors (Hoffmann et al., 2007; Liu and Roble, 2002), which will be discussed in Section 3.4.

After the central date, the winds turn westerly between $10^{-1}$ and $10^{-3} \mathrm{hPa}$ for about one week before the mesosphere starts reestablishing at higher altitudes (around $10^{-3} \mathrm{hPa}$ ). Although the easterly winds in the upper stratosphere and the westerly winds in the mesosphere remain only for about one week, the winds still differ significantly at the end of the investigated time period compared to its beginning. 

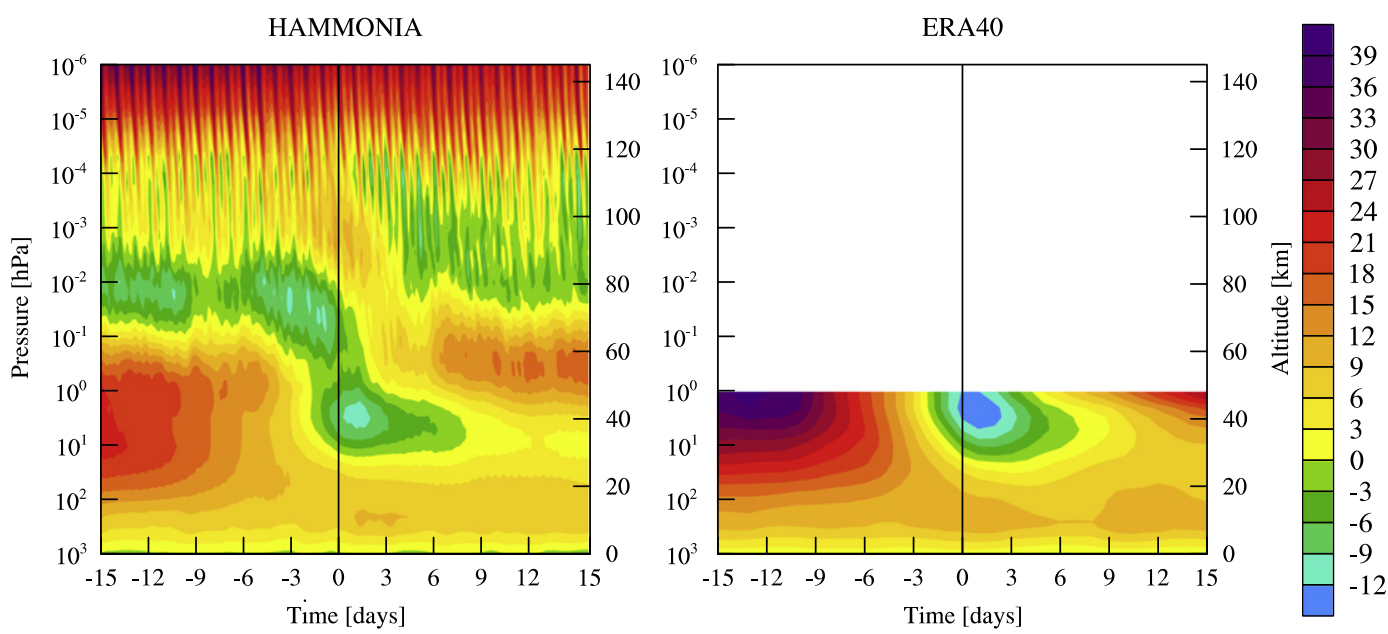

Fig. 1. Zonal mean zonal wind at $60^{\circ} \mathrm{N}$ in $\left(\mathrm{ms}^{-1}\right)$. The vertical black line indicates the central date of the SSW. Composites of (a) HAMMONIA and (b) ERA40 SSWs.
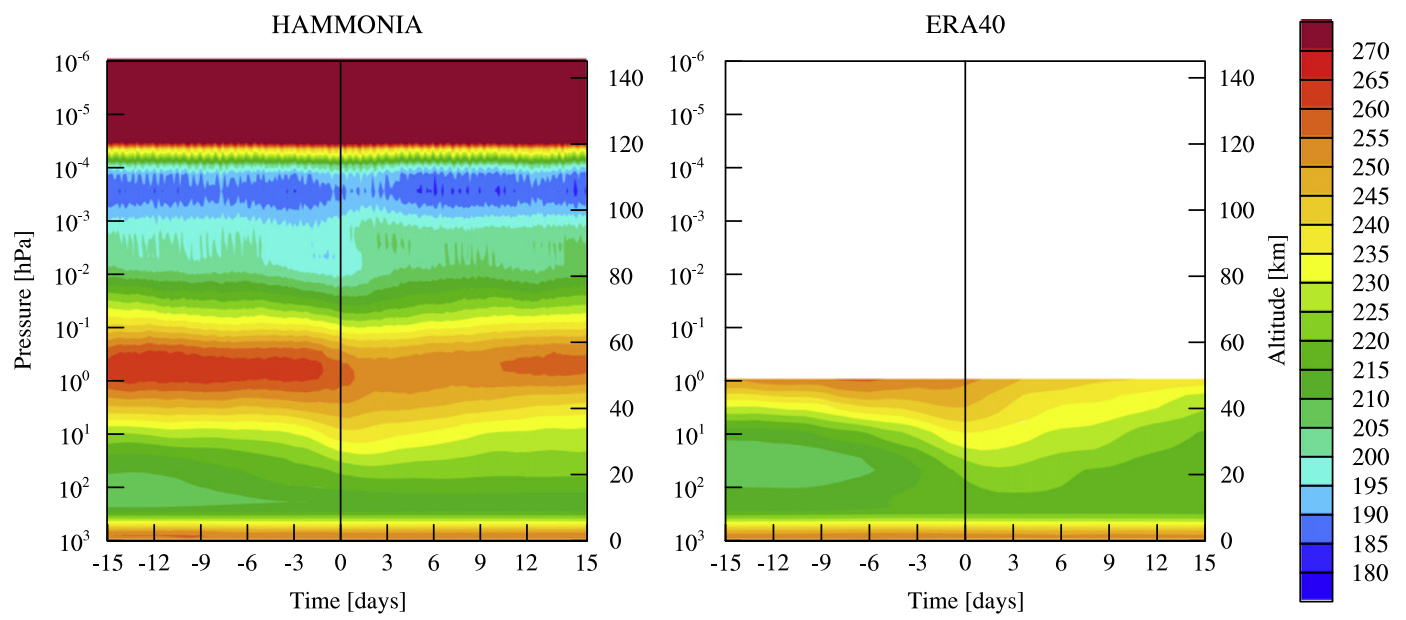

Fig. 2. Zonal mean temperature at $80^{\circ} \mathrm{N}$ in (K). The vertical black line indicates the central date of the SSW. Composites of (a) HAMMONIA and (b) ERA40 SSWs.

Above $10^{-3} \mathrm{hPa}$, the winds are dominated by tides which HAMMONIA reproduces realistically (Achatz et al., 2008). However, as we do not analyze the possible influences of SSWs on tidal activity in this paper, we compute daily mean values for the analysis in the next sections.

Fig. 2 depicts zonal average temperature for (a) HAMMONIA and (b) ERA40 at $80^{\circ} \mathrm{N}$, because the warming effects are stronger and easier to interpret near the North Pole. In both datasets the warmer temperatures of the upper stratosphere (between $10 \mathrm{hPa}$ and $1 \mathrm{hPa}$ ) start to propagate downwards several days before the central date. After the central date, the stratospheric temperatures recover. However, the upper stratospheric levels (around $10 \mathrm{hPa}$ ) remain between $5 \mathrm{~K}$ and $10 \mathrm{~K}$ warmer at the end of the analyzed period compared to day one of the composite. Despite these similarities, the reanalysis shows much steeper isotherms than the composite of the model events. Thus, the signals of the SSWs in the wind and the temperature fields are larger in the ERA40 data than in the model simulation. The vertical temperature gradient between 10 and $10^{-3} \mathrm{hPa}$ becomes significantly smaller after the central date than during the first week of the event. This feature coincides with the model study of Liu and Roble (2002). However, the stratopause is still apparent just above $1 \mathrm{hPa}$. Therefore, we can neither identify the ill-defined stratopause during the event, nor the recovery of the new stratopause at higher levels (around $10^{-2} \mathrm{hPa}$ ), as suggest by Manney et al. (2008). The tidal influence becomes obvious again at about $10^{-3} \mathrm{hPa}$.
Comparison of our dataset to characteristic numbers and to the zonal mean quantities of the ERA40 dataset (Charlton and Polvani, 2007 and Figs. 1 and 2), shows differences between observations and the model experiment. In particular, the intensity of SSWs appears to be underestimated in HAMMONIA. However, the number of events, the ratio of splits to displacements and the overall structure of winds and temperatures during the events agree fairly well. For that reason, further investigation of the HAMMONIA experiment to study vertical coupling processes and climate variability during SSWs is well justified.

\subsection{Composite analysis}

In this section, we analyze the mean anomalies of the composites over the 31 days period around the central date, which where calculated as described in Section 2.2. As explained in Section 3.1, winds are presented for $60^{\circ} \mathrm{N}$ and temperatures for $80^{\circ} \mathrm{N}$. The shaded areas in Figs. 3a and 4a indicate that the composite anomalies differ by more than one standard deviation from the model climatology. Therefore, they describe the climate variability of the different model realizations of SSWs. Applying the variability criterion to other investigated quantities led to much smaller shaded areas hinting at even higher differences between the single events (not shown). 
Zonal Wind

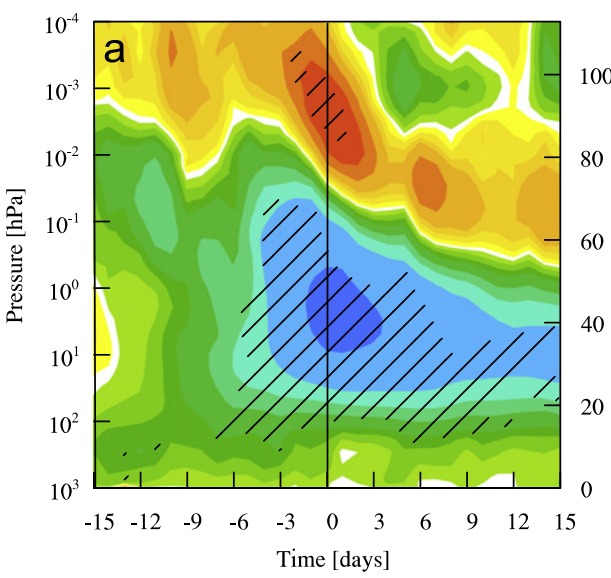

$\mathrm{du} / \mathrm{dt}$, merd. adv.

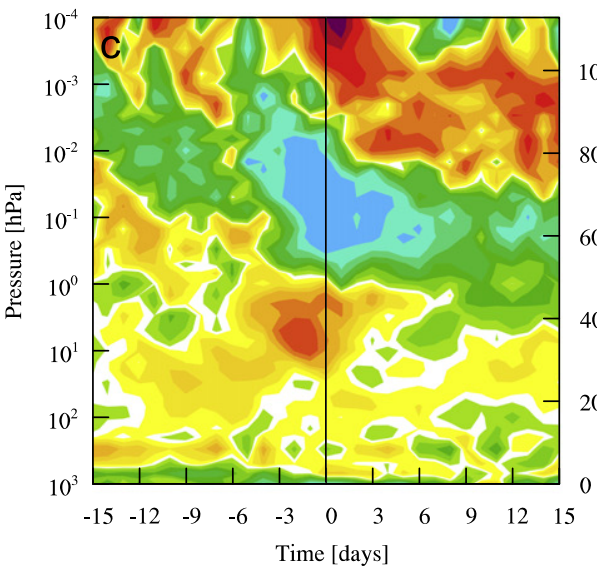

Gravity wave drag (GWD)

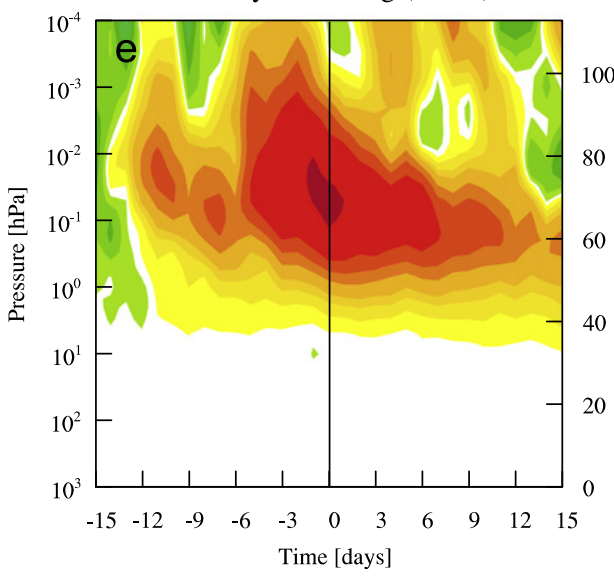

EP-Flux divergence

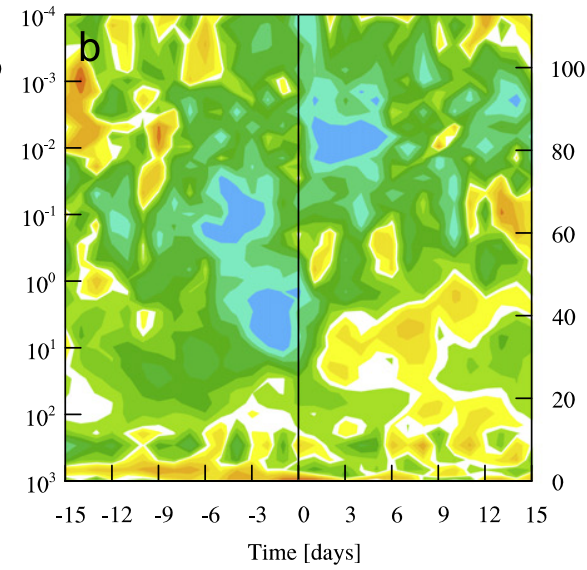

du/dt, vert. adv.

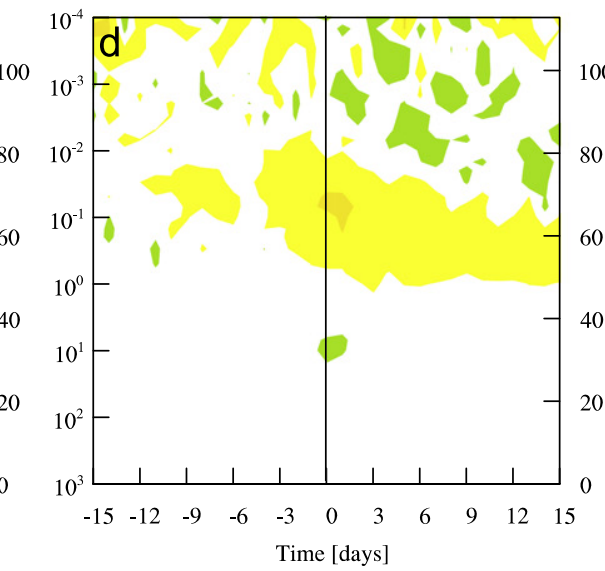

Total Wind Forcings

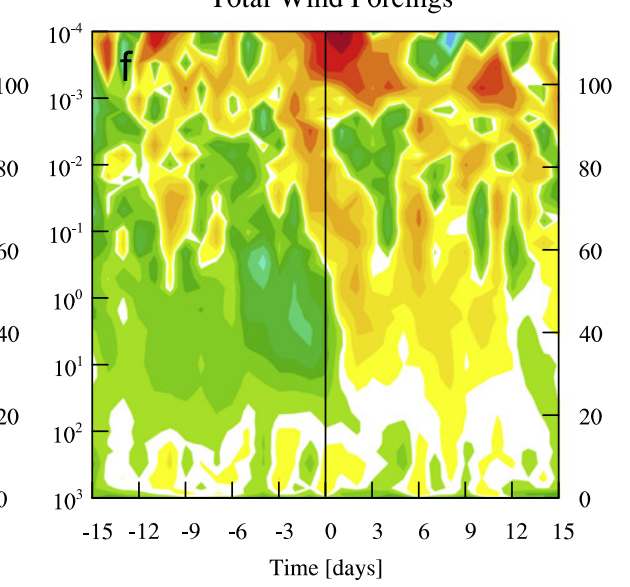

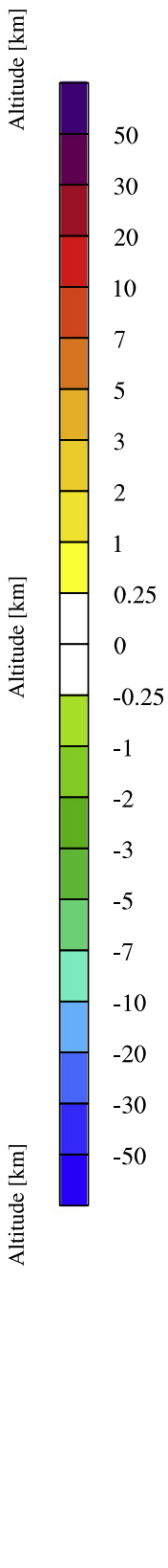

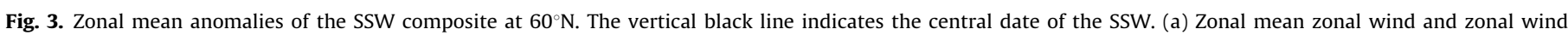

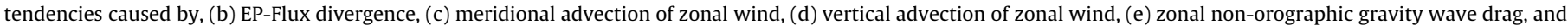

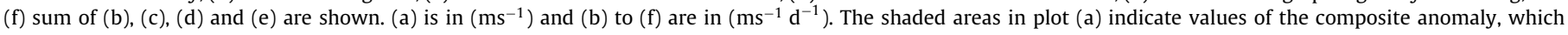
deviate from the climatological winter by more than one standard deviation.

In the stratosphere (between 100 and $1 \mathrm{hPa}$ ), Fig. 3a shows negative zonal wind anomalies of at least 3 to $5 \mathrm{~ms}^{-1}$ almost over the entire analyzed time span. On average, the negative windspeed anomalies develop first in the troposphere and in the mesosphere (between 1 and $10^{-2} \mathrm{hPa}$ ). However, these signals are relatively small and very variable from event to event. Observational data would be necessary to confirm these features.

The strongest negative anomaly of the zonal wind lies, as expected from former studies (Yoden et al., 1999), at upper stratospheric altitudes (between 10 and $1 \mathrm{hPa}$ ) around the central date and peak at values of less than $-10 \mathrm{~ms}^{-1}$. Note that the negative anomalies persist until the end of the 31-day period, which suggests a slow recovery of the atmosphere after a SSW event. Above these negative values, associated with the stratospheric wind reversal, positive values of similar magnitude indicate the remarkable changes in the mesosphere during SSWs. As for the negative values, it appears that the anomalies develop at higher altitudes and propagate downwards as time advances as 

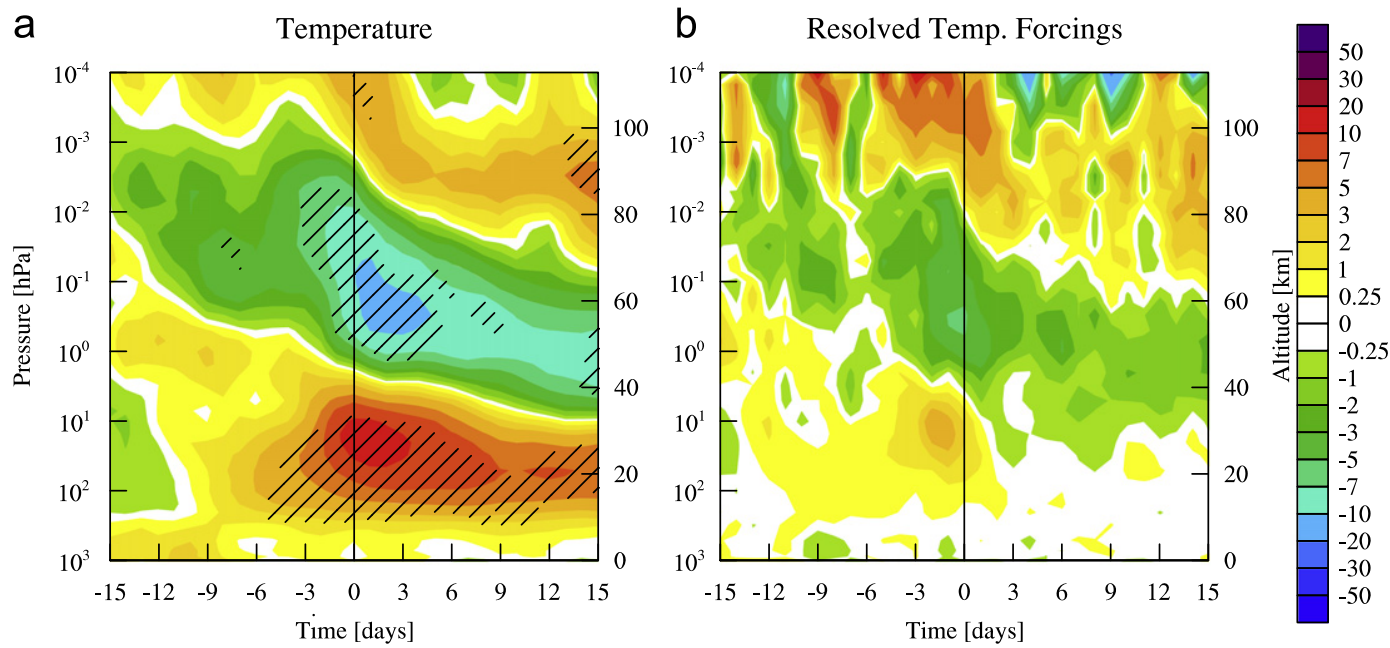

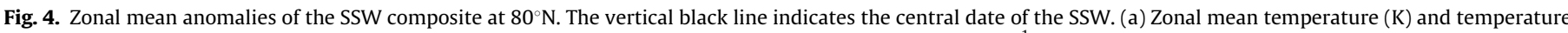

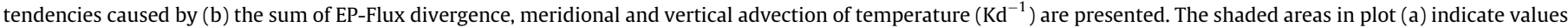
of the composite anomaly, which deviate from the climatological winter by more than one standard deviation.

previously described by Plumb and Semeniuk (2003). The positive zonal wind anomalies descend from approximately $10^{-4} \mathrm{hPa}$ at the beginning of the depicted period to less than $10^{-2} \mathrm{hPa}$ at its end. The shading of the two extrema indicates the consistency of these features in HAMMONIA.

The processes leading to the changes in zonal wind speeds are investigated by analyzing the zonal wind tendencies due to parameterized gravity waves, resolved planetary waves as well as advection of zonal mean flow. All tendencies are given in $\mathrm{ms}^{-1} \mathrm{~d}^{-1}$. The deposition of zonal momentum caused by gravity waves (from now on also referred to as 'gravity wave drag') in Fig. 3e is part of the model output. The effects of waves, which can be resolved in the model resolution, are obtained by a TEM analysis. Our TEM algorithm also calculates the meridional and vertical advection of zonal wind, which are shown in Fig. 3c,d respectively.

The influence of the vertical advection term peaking at values of about $1 \mathrm{~ms}^{-1} \mathrm{~d}^{-1}$ during the central date at about $10^{-1} \mathrm{hPa}$ is negligible. The EP-Flux divergence (Fig. $3 \mathrm{~b}$ ) describes the momentum deposition of large scale waves on the zonal mean flow. The large negative anomalies of less than $-10 \mathrm{~ms}^{-1} \mathrm{~d}^{-1}$ between 100 and $10^{-1} \mathrm{hPa}$ prior to the central date reflect the widely accepted theory that planetary waves are the primary cause of SSWs (Matsuno, 1971). However, little is known about the vertical extension of the westward acceleration due to large scale waves. In HAMMONIA, the negative anomalies due to EP-Flux divergence propagate upward with time reaching altitudes of more than $10^{-3} \mathrm{hPa}$. Recent studies have mentioned the possibility of in-situ production of planetary waves due to inhomogeneous gravity wave breaking in the middle atmosphere (Yamashita et al., 2010). Our model setup is not adequate to confirm these results, but the large EP-Flux divergence at high levels supports the idea of planetary waves playing an important role for the dynamics of the upper mesosphere and lower thermosphere (MLT region) during SSWs. At altitudes below $10 \mathrm{hPa}$, positive EP-Flux divergence acts toward the recovery of the circulation during the second half of the 31-day period.

Due to the large changes in the zonal winds, the meridional advection (Fig. 3c) also contributes substantially to the zonal wind tendencies. On average, positive anomalies counteract large parts of the negative anomalies of EP-Flux divergence before the central date and below $10^{-1} \mathrm{hPa}$. Both forcings, EP-Flux divergence and meridional advection, are of the same order of magnitude and therefore balance each other almost completely.
In the upper mesosphere negative anomalies propagate downwards from a layer between $10^{-1} \mathrm{hPa}$ and $10^{-3} \mathrm{hPa}$ to levels as low as $1 \mathrm{hPa}$ at the end of the investigated period. This longlasting negative anomaly contributes in particular to the slow recovery of the zonal winds. The relative importance of meridional advection and EP-Flux divergence can be estimated from Fig. 3f, where the sum of the three resolved forcings (Fig. 3b-d) and the gravity wave drag (Fig. 3e) is plotted. As the gravity wave forcing is negligible below $1 \mathrm{hPa}$, the total wind forcing (Fig. 3f) mainly reflects the sum of EP-Flux divergence and meridional advection for these layers. The negative total forcing before the central date, which causes the negative zonal wind anomaly, is thereby due to EP-Flux divergence. After the central date, both meridional advection and planetary wave forcing contribute to the reestablishment of the zonal winds. The major changes in zonal wind speed also strongly affect the momentum deposition of gravity waves. As gravity waves usually break in the mesosphere, they play an important part in establishing the circulation at altitudes between approximately $1 \mathrm{hPa}$ and $10^{-3} \mathrm{hPa}$. The anomaly of the resulting forcing on the zonal mean flow (Fig. 3e) shows large positive values of up to $20 \mathrm{~ms}^{-1} \mathrm{~d}^{-1}$ at levels right above the negative wind anomalies at about $10^{-1} \mathrm{hPa}$. This is due to a change in the filtering of gravity waves. The wind reversal allows for more eastward gravity waves to propagate into the mesosphere causing the change in the gravity wave forcing (Liu and Roble, 2002). The summarizing plot in Fig. 3f confirms that, on average, the gravity wave drag and the meridional advection are responsible for the large positive anomalies in the mesospheric circulation in Fig. 3a.

As a result, the negative anomalies in the zonal wind before the central date and below $1 \mathrm{hPa}$ can be explained by planetary wave activity reflected by large EP-Flux divergence. Above $10^{-1} \mathrm{hPa}$, the forcing of EP-Flux divergence, meridional advection and gravity wave forcing overlap. The positive zonal wind anomaly around $10^{-2} \mathrm{hPa}$ is mainly driven by meridional advection, but the gravity wave drag contributes as well. The small total forcing between 100 and $1 \mathrm{hPa}$ after the central date explains the slow recovery of the upper stratosphere and the lower mesosphere.

Fig. 4a shows the temperature anomalies at $80^{\circ} \mathrm{N}$ accompanying the changes in zonal wind. The characteristic features of a stratospheric warming and a mesospheric cooling above can be clearly identified. The largest positive anomaly (between $10 \mathrm{~K}$ and $20 \mathrm{~K}$ ) occurs at about $10 \mathrm{hPa}$. Above the stratopause ( $\approx 1 \mathrm{hPa})$, 
the sign of the anomalies changes indicating the occurrence of a mesospheric cooling, which is of comparable magnitude as the warming below. Both the stratospheric warming and the mesospheric cooling are shaded indicating consistency among the composite members. The thermospheric warming above approximately $10^{-2} \mathrm{hPa}$ shows a much smaller amplitude of only 3-5 K and is also more variable among the different events resulting in smaller shaded areas. The high variability will also be discussed in Section 3.4. As described for the wind anomalies, the SSWs disturb the atmosphere for more than 2 weeks, leading to significant deviations from the climatological mean over the displayed period after the central date.

The investigation of all temperature tendency terms leads to the conclusion that the only major contribution to the described scheme results from resolved forcing terms, which are obtained by the TEM analysis. Unlike for the zonal winds, the effect of gravity waves on the temperature anomalies is very small (not shown). Therefore, Fig. 4b depicts the sum of the resolved forcing terms: meridional and vertical advection as well as the temperature effect of EP-Flux divergence. However, the vertical advection by far dominates the summarizing plot indicating that adiabatic warming and cooling are the most important processes to explain the temperatures during SSWs. The resolved forcing shows three distinct layers causing the three different temperature responses.

The anomalies shown in this section support the idea of mesospheric coolings and thermospheric warmings consistently accompanying SSW events independent of the observational site or the specific event. The largest part of these anomalies is due to vertical advection. The zonal wind changes, which go along with the temperature anomalies are generated by a relatively complex interaction of meridional advection, EP-Flux divergence and momentum deposition of gravity waves.

\subsection{Split vs. displacement}

SSWs are commonly divided into splits and displacements (Charlton and Polvani, 2007; Yoden et al., 1999). These types have been defined in other studies using planetary wave amplitudes or different forms of vorticity. As we discuss in Sections 3.2 and 3.4, the variability of SSWs in our model is high. Categorizing the events can therefore help to understand the large differences among the single occurrences. Our algorithm is based on geopotential height, which is part of the standard output of GCMs. The 19 detected SSWs of this study are separated into nine splits and 10 displacements (see Table 1 ).

Fig. 5 shows the differences between the composites of splits and displacements for variables discussed in Section 3.2. Zonal wind anomalies (Fig. 5a) and the anomaly of the total forcing on zonal winds (Fig. $5 \mathrm{c}$ ) are plotted as zonal mean values at $60^{\circ} \mathrm{N}$. Temperature anomalies (Fig. 5b) and anomalies of temperature tendencies (Fig. 5d) are analyzed as zonal means at $80^{\circ} \mathrm{N}$. Shaded areas mark the 0.95 confidence interval for statistical significant differences between the
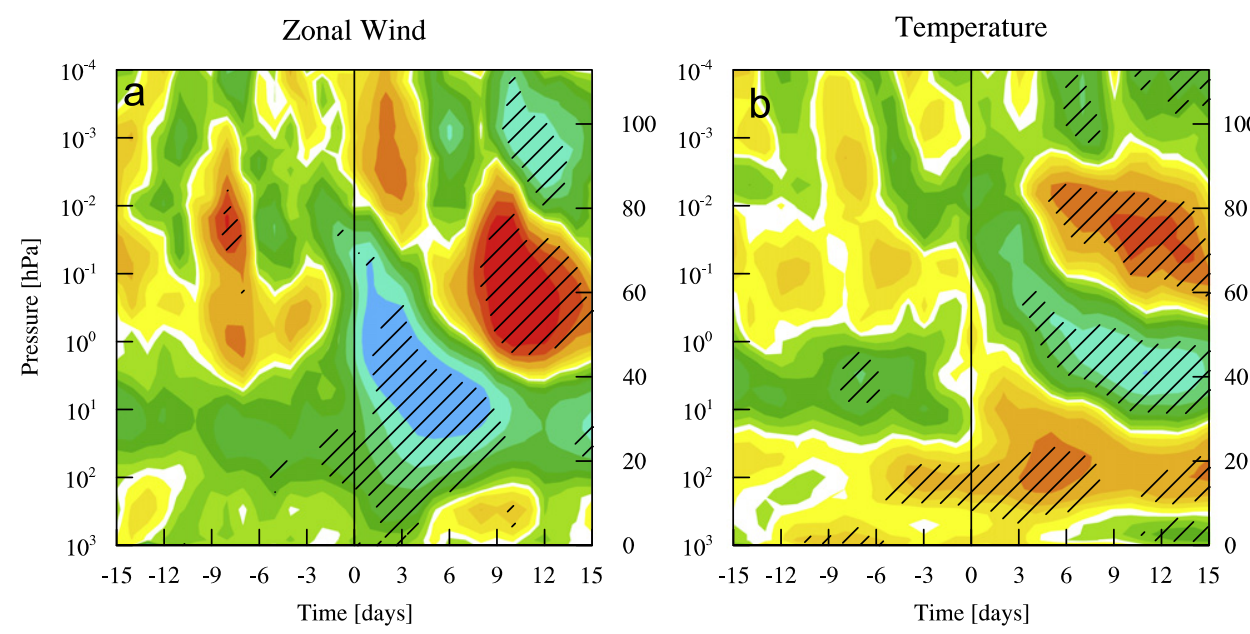

Total Wind Forcings

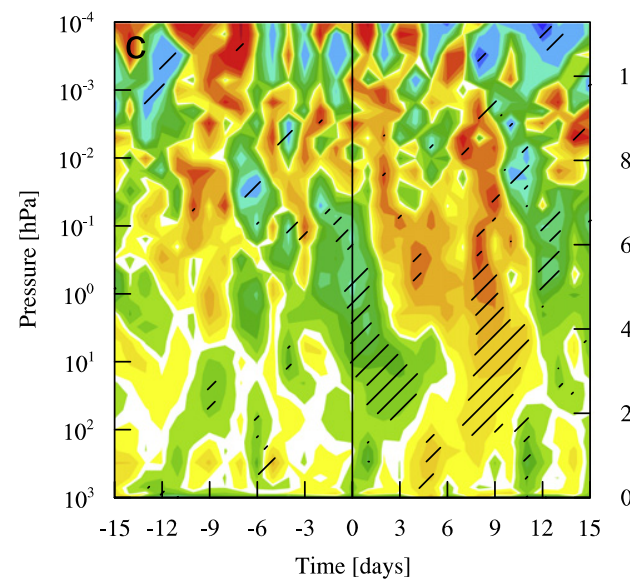

Resolved Temp. Forcings

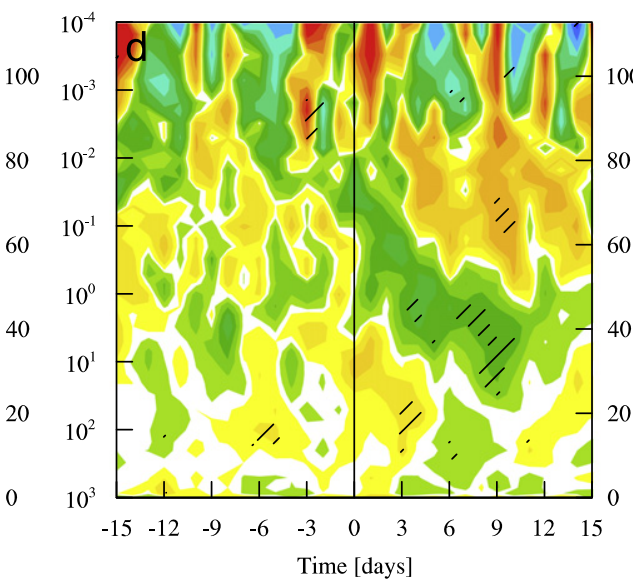

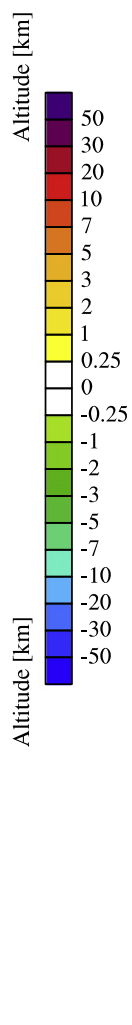

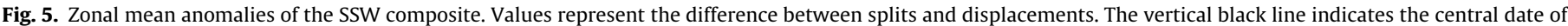

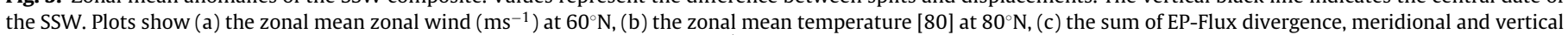

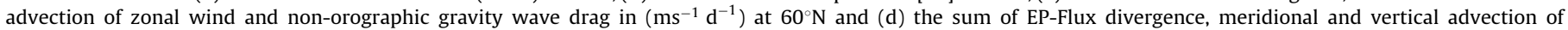
temperature $\left(\mathrm{K} \mathrm{d}^{-1}\right)$ at $80^{\circ} \mathrm{N}$. 
two types of events. All four plots indicate significant differences between the two types of events mainly after the central date suggesting the largest differences during the recovery phase of the composite SSW. The mesospheric precursors (see Section 3.4) seem to be independent from the type of event, as the zonal wind anomalies before the central date are similar for both cases between $1 \mathrm{hPa}$ and $10^{-2} \mathrm{hPa}$. The analysis indicates that in general splits are 'stronger' events, as wind and temperature anomalies are larger from the troposphere to the thermosphere. After the central date, the negative stratospheric (between 100 and $1 \mathrm{hPa}$ ) wind anomalies are more distinct during splits than during displacements leading to differences of more than $10 \mathrm{~ms}^{-1}$ for more than one week. Starting at day 24 of the investigated period, stronger mesospheric changes during split events appear as a significant positive difference. From $1 \mathrm{hPa}$ to about $10^{-2} \mathrm{hPa}$, the differences peak at values of more than $10 \mathrm{~ms}^{-1}$. At higher altitudes (above $10^{-3} \mathrm{hPa}$ ), the differences between splits and displacements are smaller, but still statistically significant.

In consistency to the described structure for zonal winds, the temperature anomalies of splits and displacements differ significantly as well. The vertical structure of warming and cooling layers discussed in Section 3.2 are more distinct during split events. The statistical significant differences take again place after the central date of the SSW composite. On average, the peak values for splits exceed the anomalies during displacements by up to $10 \mathrm{~K}$. The significant differences in zonal mean winds and temperatures between split and displacements agree with Charlton and Polvani (2007). However, it is important to note that Coughlin and Gray (2009) found no important differences between the two types of events. Their $k$-means cluster analysis suggested all events being part of a continuum of warming events.

The differences between splits and displacements can be traced back to the different dynamical processes as described in Section 3.2. Fig. $5 c$ depicts the difference in total wind forcing and can therefore be compared to Fig. 3f. The different mechanisms leading to temperature changes in the model are summarized in Fig. 5d, which was calculated similar to Fig. 4b. As expected, stronger forcings for both zonal wind and temperature reflect the described discrepancies between the 2 types of events. Fig. 5c shows a larger deceleration of the zonal mean flow during splits around the central date at a broad band of vertical levels from about $10 \mathrm{hPa}$ to $10^{-2} \mathrm{hPa}$. From day 19 to 28 of the composite, the sign of the signal changes indicating a stronger eastward acceleration from the troposphere to about $10^{-2} \mathrm{hPa}$. Large parts of these differences are statistically significant suggesting consistently different evolutions of the types of SSWs in HAMMONIA.

The described temperature differences between splits and displacements are relatively well explained by the resolved forcings (Fig. 5d). However, the additional warming in the stratosphere and cooling in the mesosphere during splits in contrast to displacements are of low significance. The dynamical processes leading to temperature differences above $1 \mathrm{hPa}$ are almost nowhere significant suggesting a high variability among the composite members. Our results suggest distinct investigations of the dynamics of the two types of events in the middle atmosphere.

\subsection{Precursors in the mesosphere}

A number of studies have investigated which circumstances usually lead to SSWs. While it is generally accepted that planetary waves propagate from the troposphere upwards initiating SSWs, there have also been discussions about mesospheric precursors, which do not cause SSWs, but might allow to predict them. Both observational (Hoffmann et al., 2002, 2007) and model studies (Liu and Roble, 2002; Plumb and Semeniuk, 2003) found that the mesospheric circulation might change several days before the actual SSW is observed. In our analysis, the anomalies of the zonal mean wind at $60^{\circ} \mathrm{N}$ and the zonal mean temperature at $80^{\circ} \mathrm{N}$ also propagate downward from mesospheric to stratospheric altitudes before the central date of the SSW composite. As many measurements are only available at specific sites, the prediction of SSWs using mesospheric precursors requires detailed knowledge about the spatial variability during the events.

We therefore decide to apply a more detailed analysis of the longitudinal distribution of zonal winds over the course of the composite event. Fig. 6 shows the zonal wind of the model composite SSWs at $60^{\circ} \mathrm{N}$ for four different longitudes to demonstrate how unequally the events develop at different locations around the globe. However, it should be noted that the winds do not only evolve differently during the SSWs, but the windspeeds differ by more than $20 \mathrm{~ms}^{-1}$ between the four locations at stratospheric altitudes between 100 and $1 \mathrm{hPa}$ at the beginning of the 31-day period. These differences do not reflect the high variability among the SSWs, but demonstrate the very asymmetric mean state of zonal winds in HAMMONIA. It is common to interpret these longitudinal differences as stationary wave structures. The choice of plots from single grid points allows better comparisons to point measurements. The composite at $15^{\circ} \mathrm{E}$ (Fig. 6a) shows a downward propagation of mesospheric easterlies to the upper stratosphere around the central date of the SSW. However, the changes are small compared to the other locations. The mesospheric winds hardly change sign between $10^{-1} \mathrm{hPa}$ and $10^{-3} \mathrm{hPa}$ and the stratospheric wind reversal occurs late and only for a short period of time around $10 \mathrm{hPa}$. The structure at $105^{\circ} \mathrm{E}$ (Fig. $6 \mathrm{~b}$ ) is more similar to the zonal average (Fig. 1a), with mesospheric winds propagating downwards earlier and causing a stronger easterly flow in the stratosphere. In contrast to Fig. 6(a) and (b), the easterly winds of the upper mesosphere (between $10^{-1} \mathrm{hPa}$ and $10^{-3} \mathrm{hPa}$ ) at $195^{\circ} \mathrm{E}$ (Fig. 6c) turn westerly about 10 days before the central date. The downward propagation of mesospheric winds appears to occur twice: between day 1 and day 8 of the SSW composite and between day 10 and the central date leading to a very long period of stratospheric easterlies. These strong easterly winds in the middle-to-upper stratosphere reflect the Aleutian High (Matthewman et al., 2009), a large anticyclonic system, which displaces the $\mathrm{NH}$ polar vortex in the boreal winter stratosphere off the pole and typically develops in the vicinity of this location. The composite mean at $285^{\circ} \mathrm{E}$ (Fig. 6d) shows easterly stratospheric winds before the central date and before the downward propagation of the mesospheric winds. However, all described changes in the mesospheric circulation (between $10^{-1} \mathrm{hPa}$ and $10^{-3} \mathrm{hPa}$ ) go along with changes at stratospheric altitudes. At all four locations in Fig. 6, the zonal winds between 100 and $1 \mathrm{hPa}$ are significantly altered before the central date of the SSW. As a result of the different mean states of the atmosphere at the beginning of the investigated period, the point in time when the stratospheric winds change their sign varies between day 8 and day 19 of the composite leading to an illdefined beginning of the event in the stratosphere. It is therefore difficult to decide whether the developments in the mesosphere are precursors to the stratospheric warming, in particular if observations from point measurements are used. Hence, different studies defined different reference dates to investigate mesospheric precursors and a process, which appears to happen before the event in one study could occur during the event in another one and consequently lead to varying conclusions about mesospheric precursors.

We changed the detection algorithm for SSWs (see Section 2.2) to test new criteria to predict the events from mesospheric observables. However, the high variability of zonal winds between $10^{-1} \mathrm{hPa}$ and $10^{-3} \mathrm{hPa}$ during the winter season led to ambiguous results. We identified some correlation to the detected SSW events, but we do not find a parameter to predict them with satisfying accuracy.

Longitudinal variations in the climatological winter as well as among single SSW events might explain the differences between 

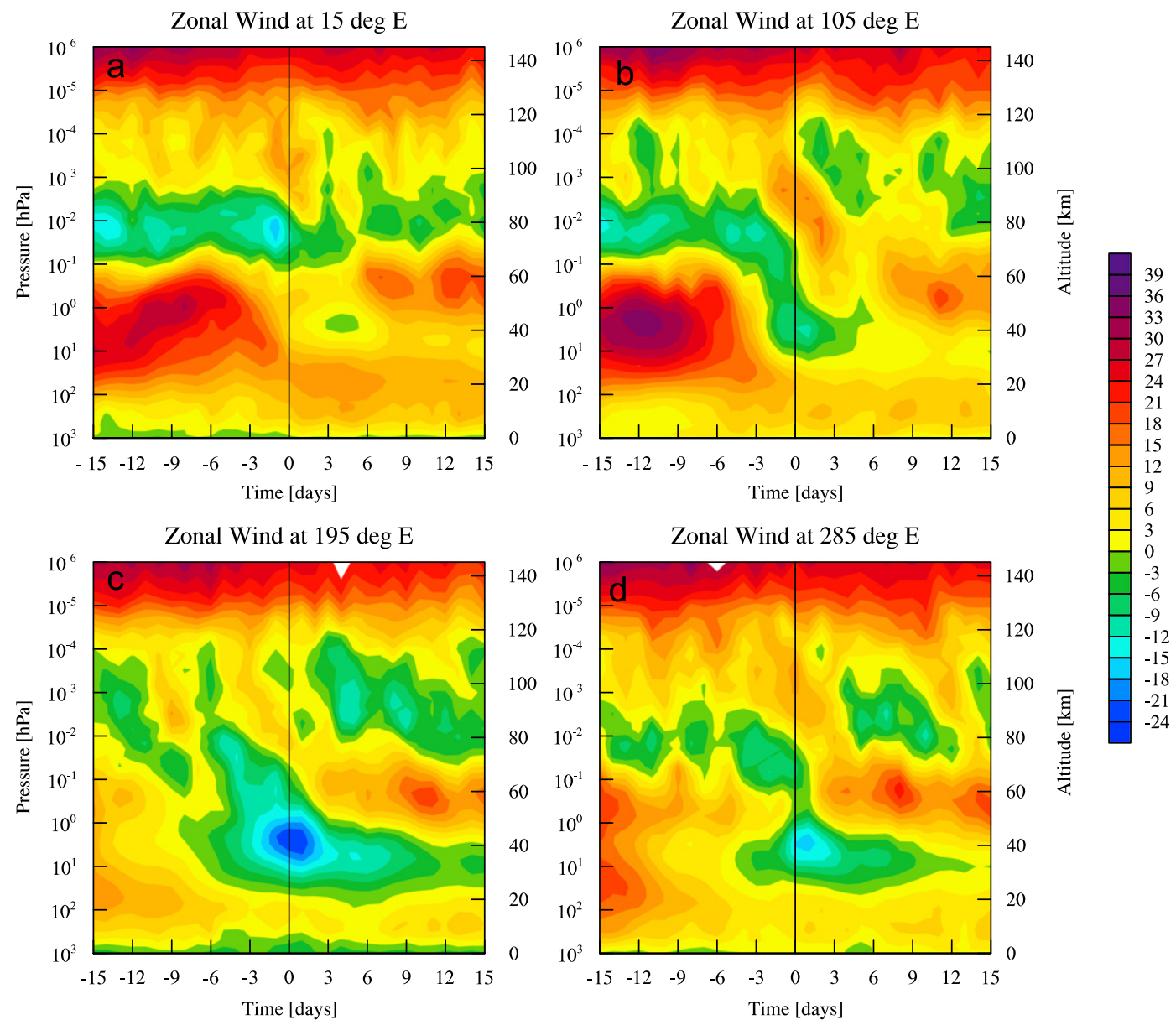

Fig. 6. Zonal wind at $60^{\circ} \mathrm{N}$ and different longitudes in $\left(\mathrm{ms}^{-1}\right)$ from the HAMMONIA composite of SSWs. The vertical black line indicates the central date of the SSW.

the point measurements and the averaged model data. The polar vortex is never exactly centered around the pole and therefore results in very different circulations at the beginning of the 31day period at the different locations. Additionally, our classification algorithm (see Appendix A) detects the average over all SSWs as a displacement with a notable Aleutian High (not shown). This asymmetry would require potential mesospheric precursors to depend on the location and the type of SSW.

As observational studies (Hoffmann et al., 2007) exploited point measurements at different locations, we investigate the zonal mean winds (Fig. 1a) and the longitudinal dependence of the mesospheric flow (Fig. 6) leading to the central date of the SSW. We find the zonal winds to be very sensitive to relatively small spatial variations. However, the described variability and the difficultly to define a mesospheric precursor remain unaffected. Furthermore, Plumb and Semeniuk (2003) stated that mesospheric precursors might also be visible in other variables, like planetary wave amplitudes or EP-flux divergence. Our composite analysis does indeed show anomalies of EP-Flux divergence at mesospheric altitudes and before the central date. But in order to define a parameter to predict SSWs, we decided to focus on variables which are accessible to observations as well.

\section{Conclusion}

The high top GCM HAMMONIA is used to investigate characteristics of the vertical coupling during SSWs. The detected number of internally produced events (19) is reasonable. Composites are built to analyze the typical features of SSWs and their variability. A comparison of the experiment climatology to the ERA40 climatology shows some deficiencies in the height of the stratopause, the strength of the zonal winds and the amplitude of SSWs. However the overall picture leads to the conclusion that the numerical representation in the model is adequate to analyze characteristics of SSWs.

In the composite mean, a mesospheric cooling of comparable amplitude accompanies the stratospheric warming. Although it is of smaller magnitude, the relatively recent reports (Liu and Roble, 2002) of thermospheric warmings could also be confirmed to be a consistent characteristic of SSWs. These temperature changes are associated with large anomalies in the zonal mean zonal wind, which result from the combined effects of planetary waves, meridional advection and gravity wave momentum deposition.

We developed an algorithm to distinguish between splits and displacements, which uses the easily accessible geopotential height as input variable. For ERA40 the classification agrees well with the results of Charlton and Polvani (2007). In HAMMONIA, there are significant differences in the zonal mean zonal wind and the zonal mean temperature between the two types of events. This result confirms findings of Charlton and Polvani (2007), but disagrees with the recent results of Coughlin and Gray (2009), who found splits and displacements to be two different members within a continuum of warming events. On average, the anomalies of the split events are up to $20 \mathrm{~ms}^{-1}$ smaller and up to $7 \mathrm{~K}$ warmer in the stratosphere than the displacements. Similar values of opposing sign were obtained for the mesosphere. The differences could be traced back to variations in the forcing quantities, which indicate a stronger negative acceleration of the zonal mean flow around the central date of splits. During 
the week after the central date, the sign of the forcing terms reverses. The results, obtained with HAMMONIA, suggest that it is reasonable to investigate splits and displacements as dynamically distinct events in the middle atmosphere. However, we did find the largest differences after the central date, so the question of mesospheric precursors seems to be independent from the type of event. It should be noted that the lack of observations above the stratopause will cause difficulties, if sample sizes are further reduced.

Although our model study confirms dynamical processes in the mesosphere to be initiated before the central date of the composite event, we do not find a persistent mesospheric precursor of SSWs as suggested by Hoffmann et al. (2007) and Liu and Roble (2002). As a result, we did not find a criterion to detect SSWs before the central date using mesospheric zonal winds. Tests with an altered detection algorithm did not lead to sufficient agreements between mesospheric processes and the onset of SSWs at $10 \mathrm{hPa}$. The plots for different longitudes at the same latitude showed that, on average, the mean circulation as well as the single events are too asymmetric to detect mesospheric precursors with point measurements. Furthermore, it is difficult to decide where the circulation changes appear first, as the stratospheric circulation is clearly affected prior to the central date of the SSW as well. However, the increasing number of satellite observations will lead to new opportunities to define mesospheric precursors. If mesospheric measurements become available with a high spatial and temporal coverage, future studies should be able to account for longitudinal differences. We conclude that, in our model, the variability of zonal winds in the mesosphere and the stratosphere prior to the central date of SSWs is too large to predict SSWs consistently. However, we can not rule out that the on average too low anomalies simulated by HAMMONIA make the identification of precursors even more difficult in the model than in reality.

Further research should compare more mesospheric observations to the presented data to ensure the validity of the conclusions. In particular, long time series of satellite data could fill the gap between point measurements and large scale models.

We focused on specific aspects of vertical coupling mechanisms during SSWs, but recent studies stated that tides might also be affected during SSWs (Fuller-Rowell et al., 2010). Our composite could also be exploited to deepen the understanding of interhemispheric coupling mechanisms over multiple layers, as has been suggested in Xu et al. (2009).

\section{Acknowledgments}

We are grateful to Christoph Zülicke for many helpful comments and discussions about unpublished results. We also thank Peter Hoffmann for providing insights to ongoing measurements and chairing the ISSI group on coupling processes in the middle and upper atmosphere. We thank Elisa Manzini, Thomas Krismer and Anna Possner for many fruitful discussions and comments during the evolution of this work. The 'Deutsche Klimarechenzentrum' (DKRZ) has performed the computations, that led to this dataset. We further thank the two reviewers who helped improve the publication significantly.

\section{Appendix A. Classification algorithm}

We categorize the events as splits and displacements as suggested by Charlton and Polvani (2007), Yoden et al. (1999) and Bancala et al. (2012). These studies use potential vorticity gradients and planetary wave number amplitudes to distinguish between the two types of events. We decided to use geopotential height for simplicity reasons.

First, the absolute minimum of the geopotential height at $10 \mathrm{hPa}, \Phi_{10}(\lambda, \phi)$, is determined and assumed to represent the center of the main polar vortex:

$\Phi_{10, \text { center }}=\min \left\{\Phi_{10}(\lambda, \phi)\right\}$

In a second step, we identify the local minima $\Phi_{10, \text { local, } i}$ on every latitude in the model grid from the North Pole to $20^{\circ} \mathrm{N}$.

The decision if a particular SSW is categorized as a split or a displacement is determined by searching for paths from the location of $\Phi_{10, \text { center }}$ to the locations of all $\Phi_{10, \text { local, } i}$ s in the latitude-longitude model grid $(\lambda, \phi)$ along positive geopotential height gradients. If our algorithm finds such a path for every local minimum, we refer to the event as a displacement. Otherwise, it is called a split.

Additionally, we introduce two adjustable parameters to the routine. The first parameter reduces the number of $\Phi_{10, \text { local, } i}$ 's to make sure that only significant minima are considered. If

$\left(\Phi_{10, \text { mean }}-\Phi_{10, \text { local }, i}\right)<0.3 \cdot\left(\Phi_{10, \text { mean }}-\Phi_{10, \text { center }}\right)$,

where $\Phi_{10 \text {,mean }}=\overline{\Phi_{10}(\lambda, \phi)}$ for $20^{\circ} \leq \phi \leq 90^{\circ}$,

the local minimum $\Phi_{10, \text { local }, i}$ is excluded from the computations.

A second parameter accounts for the finite resolution in model and reanalysis data. It allows for path steps $p$ with slightly negative gradients. We set it to allow for

$p>-0.03 \cdot\left(\Phi_{10 \text {,mean }}-\Phi_{10, \text { center }}\right)$.

These parameter settings are arbitrary but are experimentally determined for the T31 horizontal grid according to our subjective impressions. For ERA40 data interpolated to T31, the classification routine produces similar results as the one presented by Charlton and Polvani (2007).

\section{References}

Achatz, U., Grieger, N., Schmidt, H., 2008. Mechanisms controlling the diurnal solar tide: analysis using a GCM and a linear model. Journal of Geophysical Research 113 (A8), A08303. URL 〈http://www.agu.org/pubs/crossref/2008 2007JA012967.shtml $>$.

Andrews, D.G., Holton, J., Leovy, C., 1987. Middle Atmosphere Dynamics, vol. 40. Academic Press.

Baldwin, M.P., Dunkerton, T.J., 2001. Stratospheric harbingers of anomalous weather regimes. Science 294 (5542), 581-584.

Bancala, S., Krüger, K., Giorgetta, M., 2012. The preconditioning of major sudden stratospheric warmings. Journal of Geophysical Research 117 (D4), D04101.

Charlton, A.J., Polvani, L.M., 2007. A new look at stratospheric sudden warmings. Part I: climatology and modeling benchmarks. Journal of Climate 20 (3), 449-469.

Charlton-Perez, A.J., Polvani, L.M., 2011. Corrigendum. Journal of Climate 24 (22), 5951. URL 〈http://dx.doi.org/10.1175/JCLI-D-11-00348.1〉.

Coughlin, K., Gray, L.J., 2009. A continuum of sudden stratospheric warmings. Journal of the Atmospheric Sciences 66 (2), 531-540.

Fuller-Rowell, T., Wu, F., Akmaev, R., Fang, T.-W., Araujo-Pradere, E., 2010. A whole atmosphere model simulation of the impact of a sudden stratospheric warming on thermosphere dynamics and electrodynamics. Journal of Geophysical Research 115, A00G08.

Goncharenko, L.P., Chau, J.L., Liu, H.-L., Coster, A.J., 2010. Unexpected connections between the stratosphere and ionosphere. Geophysical Research Letters 37 (10), 1-6.

Hines, C., 1997a. Doppler-spread parameterization of gravity-wave momentum deposition in the middle atmosphere. Part 1: basic formulation. Journal of Atmospheric and Solar-Terrestrial Physics 59 (4), 371-386.

Hines, C., 1997b. Doppler-spread parameterization of gravity-wave momentum deposition in the middle atmosphere. Part 2: broad and quasi monochromatic spectra, and implementation. Journal of Atmospheric and Solar-Terrestrial Physics 59 (4), 387-400.

Hoffmann, P., Singer, W., Keuer, D., 2002. Variability of the mesospheric wind field at middle and Arctic latitudes in winter and its relation to stratospheric circulation disturbances. Journal of Atmospheric and Solar-Terrestrial Physics 64 (8-11), 1229-1240.

Hoffmann, P., Singer, W., Keuer, D., Hocking, W., Kunze, M., Murayama, Y., 2007. Latitudinal and longitudinal variability of mesospheric winds and temperatures during stratospheric warming events. Journal of Atmospheric and SolarTerrestrial Physics 69 (17-18), 2355-2366. 
Kinnison, D.E., Brasseur, G.P., Walters, S., Garcia, R.R., Marsh, D.R., Sassi, F., Harvey, V.L., Randall, C.E., Emmons, L., Lamarque, J.F., Hess, P., Orlando, J.J., Tie, X.X. Randel, W., Pan, L.L., Gettelman, A., Granier, C., Diehl, T., Niemeier, U., Simmons, A.J., 2007. Sensitivity of chemical tracers to meteorological parameters in the MOZART-3 chemical transport model. Journal of Geophysical Research 112 (D20)), 1-24.

Liu, H.-L., Roble, R.G., 2002. A study of a self-generated stratospheric sudden warming and its mesospheric-lower thermospheric impacts using the coupled TIME-GCM/CCM3. Journal of Geophysical Research 107 (D23), 1-18.

Lott, F., Miller, M., 1997. A new subgrid-scale orographic drag parameterization: its formulation and testing. Quarterly Journal of the Royal Meteorological Society 123 (537), 101-127.

Manney, G.L., Krüger, K., Pawson, S., Minschwaner, K., Schwartz, M.J., Daffer, W.H. Livesey, N.J., Mlynczak, M.G., Remsberg, E.E., Russell, J.M., Waters, J.W., 2008 The evolution of the stratopause during the 2006 major warming: satellite data and assimilated meteorological analyses. Journal of Geophysical Research 113 (D11), 1-16.

Manzini, E., Giorgetta, M.A., Esch, M., Kornblueh, L., Roeckner, E., 2006. The influence of sea surface temperatures on the northern winter stratosphere: ensemble simulations with the MAECHAM5 model. Journal of Climate 19 (16), 3863.

Matsuno, T., 1970. Vertical propagation of stationary planetary waves in the winter Northern Hemisphere. Journal of the Atmospheric Sciences 27 (6), 871-883.

Matsuno, T., 1971. A dynamical model of the stratospheric sudden warming. Journal of Atmospheric Sciences 28, 1479-1494.

Matthewman, N.J., Esler, J.G., Charlton-Perez, A.J., Polvani, L.M., 2009. A new look at stratospheric sudden warmings. Part III: polar vortex evolution and vertical structure. Journal of Climate 22 (6), 1566-1585. URL < http://journals.ametsoc. org/doi/abs/10.1175/2008JCLI2365.1 >.

Plumb, R.A., Semeniuk, K., 2003. Downward migration of extratropical zonal wind anomalies. Journal of Geophysical Research 108 (D7), 4223.

Polvani, L.M., 2002. Tropospheric response to stratospheric perturbations in a relatively simple general circulation model. Geophysical Research Letters 29 (7), 40-43.
Roeckner, E., Brokopf, R., Esch, M., Giorgetta, M., Hagemann, S., Kornblueh, L., Manzini, E., Schlese, U., Schulzweida, U., 2006. Sensitivity of simulated climate to horizontal and vertical resolution in the ECHAM5 atmosphere model. Journal of Climate 19 (16), 3771.

Scherhag, R., 1952. Die explosionsartigen Stratosphärenerwärmungen des Spätwinters 1951/52. Berichte des Deutschen Wetterdienstes der US-Zone 6 (38), 51-63.

Schmidt, H., Brasseur, G.P., Charron, M., Manzini, E., Giorgetta, M.A., Diehl, T., Fomichev, V.I., Kinnison, D., Marsh, D., Walters, S., 2006. The HAMMONIA chemistry climate model: sensitivity of the mesopause region to the 11-year solar cycle and $\mathrm{CO}_{2}$ doubling. Journal of Climate 19 (16), 3903.

Schmidt, H., Brasseur, G.P., Giorgetta, M.A., 2010. Solar cycle signal in a general circulation and chemistry model with internally generated quasi-biennial oscillation. Journal of Geophysical Research 115, 1-16.

Siskind, D.E., Eckermann, S.D., Coy, L., McCormack, J.P., Randall, C.E., 2007. On recent interannual variability of the Arctic winter mesosphere: implications for tracer descent. Geophysical Research Letters 34 (9), 1-5.

Smith, A.K., 1996. Longitudinal variations in mesospheric winds: evidence for gravity wave filtering by planetary waves. Journal of the Atmospheric Sciences 53 (8), 1156-1173.

Wilks, D.S. 2006. Statistical Methods in the Atmospheric Sciences, 2nd ed. vol. 91. Academic Press.

Xu, X., Manson, A.H., Meek, C.E., Chshyolkova, T., Drummond, J.R., Hall, C.M., Riggin, D.M., Hibbins, R.E., 2009. Vertical and interhemispheric links in the stratosphere-mesosphere as revealed by the day-to-day variability of AuraMLS temperature data. Annales Geophysicae 27 (9), 3387-3409.

Yamashita, C., Liu, H.-L., Chu, X., 2010. Responses of mesosphere and lower thermosphere temperatures to gravity wave forcing during stratospheric sudden warming. Geophysical Research Letters 37 (9), 1-5.

Yoden, S., Yamaga, T., Pawson, S., Langematz, U., 1999. A composite analysis of the stratospheric sudden warmings simulated in a perpetual January integration of the Berlin TSM GCM. Journal-Meteorological Society of Japan 77 (2), 431-445. 